\title{
Spinal Cord Stimulation for Painful Diabetic Peripheral Neuropathy: A Systematic Review
}

\author{
Josianna V. Henson · Narayana C. Varhabhatla · Zvonimir Bebic · \\ Alan D. Kaye · R. Jason Yong · Richard D. Urman · Justin S. Merkow
}

Received: May 11, 2021 / Accepted: June 15, 2021 / Published online: July 10, 2021

(C) The Author(s) 2021

\begin{abstract}
Painful diabetic neuropathy is a common disease that results in significant pain and disability. Treatment options have traditionally consisted of conservative measures including topical and oral medication management as well as transcutaneous electrical stimulation units. These treatments demonstrate various degrees of efficacy, and many times initial treatments are discontinued, indicating low levels of satisfaction or poor tolerability. Spinal
\end{abstract}

Supplementary Information The online version contains supplementary material available at https:// doi.org/10.1007/s40122-021-00282-9.

J. V. Henson · N. C. Varhabhatla · Z. Bebic .

J. S. Merkow $(\bowtie)$

Department of Anesthesiology, University of Colorado School of Medicine, Aurora, CO 80045,

USA

e-mail: justin.merkow@cuanschutz.edu;

justin.merkow@gmail.com

R. J. Yong · R. D. Urman

Department of Anesthesiology, Perioperative and Pain Medicine, Brigham and Women's Hospital, Boston, MA, USA

A. D. Kaye

Department of Anesthesiology, Louisiana Statement

University, New Orleans, LA, USA cord stimulation has been proposed as an alternative therapy for treatment of painful diabetic neuropathy of the lower extremities. We performed a systematic literature review to evaluate the safety and effectiveness of this procedure. A literature search identified 14 prospective studies. Based on our analysis of the available evidence, there is moderate-quality evidence for the safety and efficacy of spinal cord stimulation for painful diabetic neuropathy. However, further high-quality research, including a large-scale randomized controlled trial is warranted.

Keywords: Diabetic peripheral neuropathy; Spinal cord stimulation; Peripheral neuropathy; Electric nerve stimulation; Neuromodulation; Diabetes; Neuropathic pain 


\section{Key Summary Points}

It is estimated that $20 \%$ of the 34 million patients in the United States with diabetes will develop diabetic peripheral neuropathy (PDN).

Treatment has traditionally consisted of medication management, lifestyle modifications, and physical therapy modalities.

There is a growing body of evidence suggesting that spinal cord stimulation is a safe and effective treatment option for patients with diabetic peripheral neuropathy.

Although it is difficult to recommend specific selection criteria based on the literature, PDN patients treated with SCS seem to be older than 50, have diabeticrelated pain greater than 5 years, have failed multiple neuropathic medications, and have an A1C less than 10.

Further high-quality studies are warranted to evaluate efficacy as well as clinical guidance regarding patient selection.

\section{DIGITAL FEATURES}

This article is published with digital features, including a summary slide to facilitate understanding of the article. To view digital features for this article go to https://doi.org/10.6084/ m9.figshare.14785575.

\section{INTRODUCTION}

Thirty-four million people in the United States have currently been diagnosed with diabetes (10.5\% of the US population), according to data from the Centers for Disease Control and Prevention. Additionally, there are 88 million people with pre-diabetes, which has resulted in
$\$ 327$ billion in health-care-related costs and lost productivity $[1,2]$. It is estimated that $20 \%$ of patients with diabetes will develop painful diabetic neuropathy (PDN) [3].

Traditionally, patients suffering from PDN are first treated conservatively with medication management. The only treatments for PDN that are approved by the US Food and Drug Administration (FDA) are pregabalin, duloxetine, and tapentadol extended-release. Gabapentin is also commonly prescribed, as are tricyclic antidepressants, opioid analgesics, topical lidocaine, capsaicin cream, isosorbide dinitrate spray, and transcutaneous electrical nerve stimulation [4]. These treatments demonstrate various degrees of success, and many times initial treatments are discontinued, indicating low levels of satisfaction or poor tolerability [5].

Therefore, there exists a need to address the treatment gap for patients suffering from diabetic peripheral neuropathy that are getting incomplete relief from conservative treatment. Neuromodulation with spinal cord stimulation has the potential to address this gap. Spinal cord stimulation has been shown to result in significant pain relief in patients with debilitating neuropathic pain conditions such as failed back surgery syndrome and complex regional pain syndrome [6-9]. The neurophysiology of how spinal cord stimulation achieves pain relief is not completely understood [10]. Both tonic and paresthesia-free (including burst and high-frequency) stimulation are able to generate highly effective and clinically significant analgesia [11-13]. There is a growing body of evidence in the literature that spinal cord stimulation is a safe and effective therapy for PDN. The goal of this review is to examine the evidence and outcomes related to spinal cord stimulation for painful diabetic peripheral neuropathy in order to further evaluate the safety and efficacy of these procedures.

\section{METHODS}

This review was completed with the assistance of a research librarian at University of Colorado Strauss Health Sciences Library, Aurora, 
Colorado. The protocol was designed according to PRISMA guidelines. Relevant literature was identified in the following bibliographic databases: Ovid MEDLINE-ALL (Ovid 1946-current), Embase (Embase.com, 1974-current), and the Cochrane Library (Wiley). No year limits were applied to the searches. The search strategies are based on the concepts of diabetic peripheral neuropathy and spinal cord stimulation using multiple subject headings and text-word terms for each concept. The following keywords were included in the search: "diabetic", "neuropathy", "polyneuropathy", "mononeuropathy", "autonomic", "neuralgia", "PDN", "peripheral diabetic", "diabetic neuropathies", "spinal", "high-frequency", "electrical stimulation", "electric stimulation therapy", "spinal cord stimulation". Supplementary Material 1 shows the full search strategies for all bibliographic databases. Searches were conducted on January 26, 2021.

Discovery of appropriate subject headings involved examining how gold standard articles are indexed, by assessing matches made by the subject heading databases (such as matches to MeSH in Medline), and by using word analysis tools specifically PubMed PubReminer (https:// hgserver2.amc.nl/cgi-bin/miner/miner2.cgi/) and TerMine (http://nactem.ac.uk/software/ termine/) [14]. All retrieved records were managed with Endnote version 9.3, a citation management application, and with Covidence (www.covidence.org), a systematic review application.

Inclusion criteria for this review include randomized controlled trials (RCTs), retrospective, case-control, and prospective observational studies assessing spinal cord stimulation for PDN in both type 1 and type 2 diabetics who were 18 years old or older. Exclusion criteria included case reports, case series, historical articles, letters, review articles, foreign language studies, non-human studies, cadaver studies, or conference abstracts.

The citations identified were assessed for inclusion in the review using a multi-stage process. Initially, two reviewers (JM and NV) independently screened all study titles and abstracts identified by the electronic searches to identify the potentially relevant articles to be retrieved. Next, full-text copies of these studies were obtained and assessed independently by two reviewers for inclusion using the previously established inclusion criteria (JH and ZB). Any disagreements were resolved through discussion at each stage, and, if necessary, in consultation with a third reviewer (JM).

This article is based on previously conducted studies and does not contain any new studies with human participants or animals performed by any of the authors.

\section{Quality Assessment}

A risk-of-bias assessment was completed for all randomized control trials using the Revised Cochrane risk-of-bias tool for randomized trials (RoB 2) [15]. Studies were assessed across multiple domains per the specifications of the RoB 2 tool. Studies found to have an overall high risk of bias were judged to be at high risk of bias in at least one domain or were judged to have some concerns for multiple domains. The prospective observational studies were not assessed with this tool, as they were considered to have an implicit risk of bias based on the nature of their study design. Results of this assessment are demonstrated in Fig. 2.

\section{RESULTS}

Our initial search resulted in 443 references, which were imported for screening. Following full-text review, 14 studies were reviewed. Of these, there were three RCTs and 11 prospective observational studies. See Fig. 1 for PRISMA flow diagram. Table 1 shows the results of the studies and describes each study that was included in the review.

\section{Randomized Controlled Studies}

de Vos et al. [21] performed the first multicenter randomized controlled trial analyzing the effectiveness of spinal cord stimulation in patients with PDN. Sixty patients with PDN of the lower extremities refractory to conventional medical therapy were randomized $2: 1$ to best 


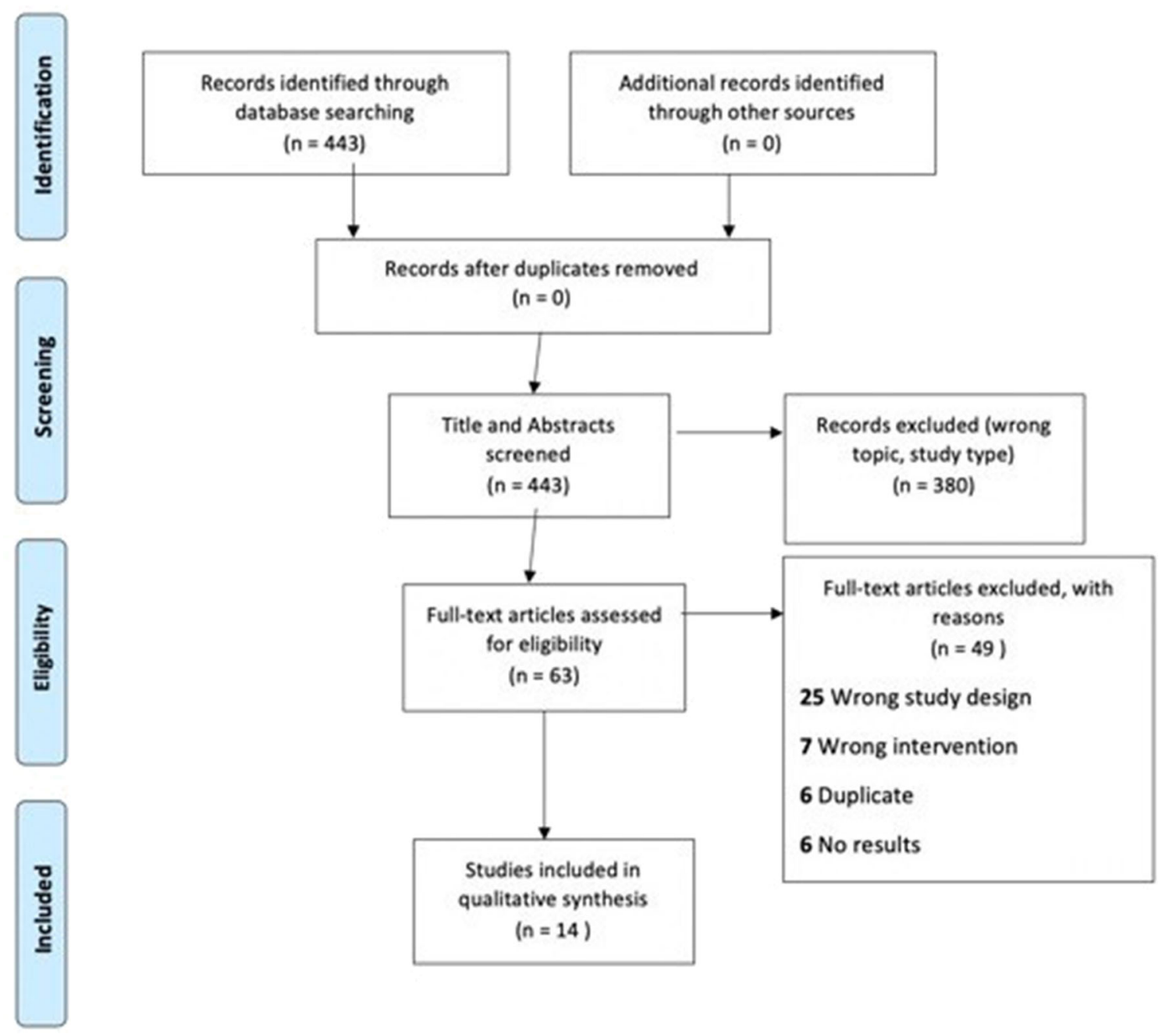

Fig. 1 PRISMA 2009 flow diagram [34]

conventional medical practice with spinal cord stimulation or without. Both groups were assessed at regular intervals over a 6-month period using the Euro-Qol 5d, short form McGill Pain Questionnaire (SF-MPQ) and a Visual Analogue Scale (VAS, ranging 0-100). The average VAS was 73 in the SCS group and 67 in the control group. After 6 months of treatment, the average VAS of the SCS group was reduced to 31 $(p<0.001)$ and was $67(p=0.97)$ in the control group. Improvements in pain, health, and quality of life were also demonstrated in the SFMPQ and the Euro-QoL 5d questionnaires. There were two adverse events related to the implantation procedure including pain due to the implanted pulse generator in two patients, as well as electrode lead migration in one patient. There was one infection during SCS trial, which resolved and was followed by a permanent implantation. One patient had previously undiagnosed coagulopathy, which complicated the implantation procedure and resulted in hospitalization; $95 \%$ of the patients in the SCS group might or would definitely recommend SCS treatment to other patients with PDN.

Slangen et al. also performed a multicenter randomized controlled trial in 36 patients with PDN of the lower limbs [22]. Twenty-two patients were randomly assigned to SCS in combination with best medical treatment while 14 were allocated to a best medical treatment 


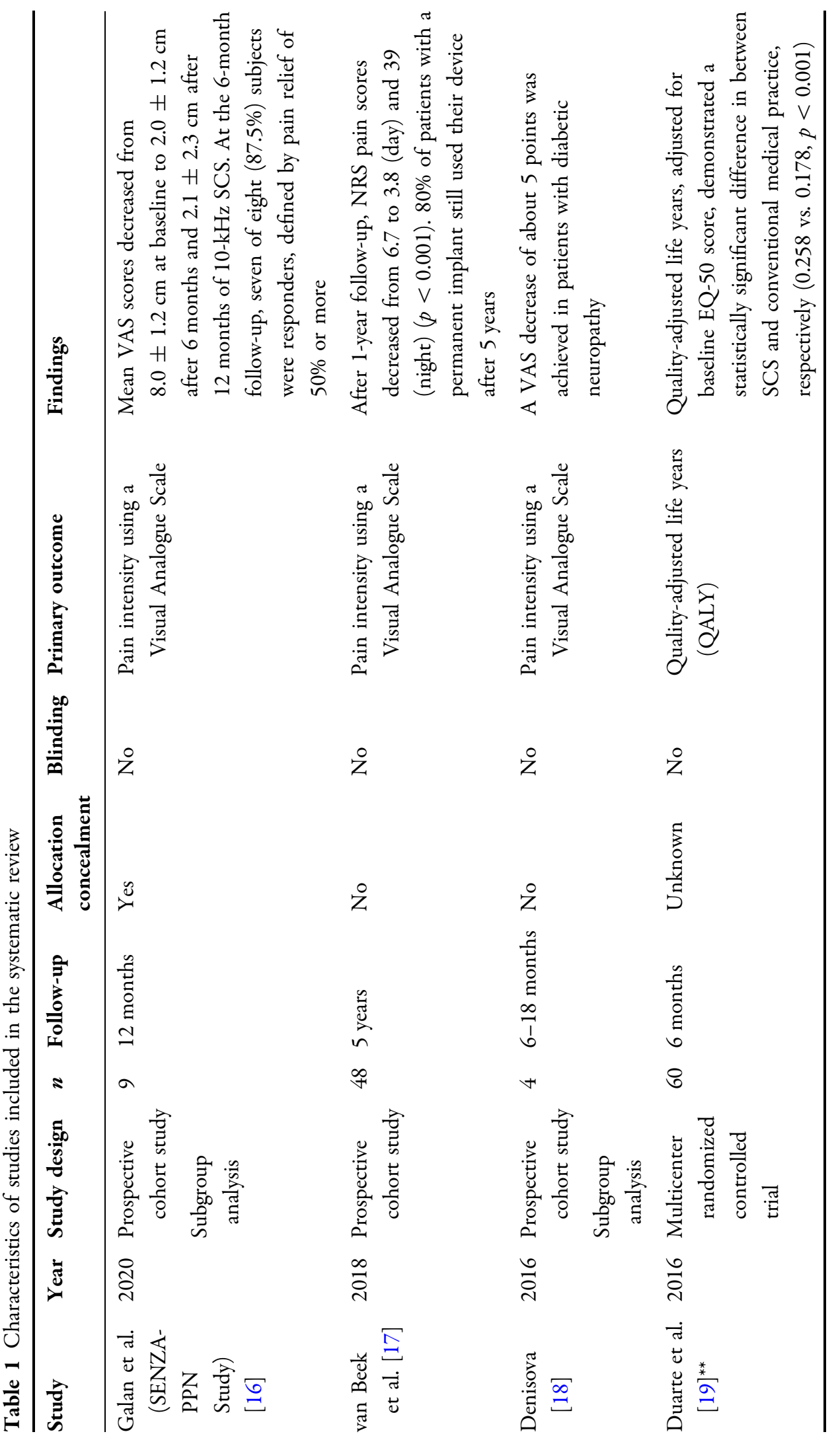




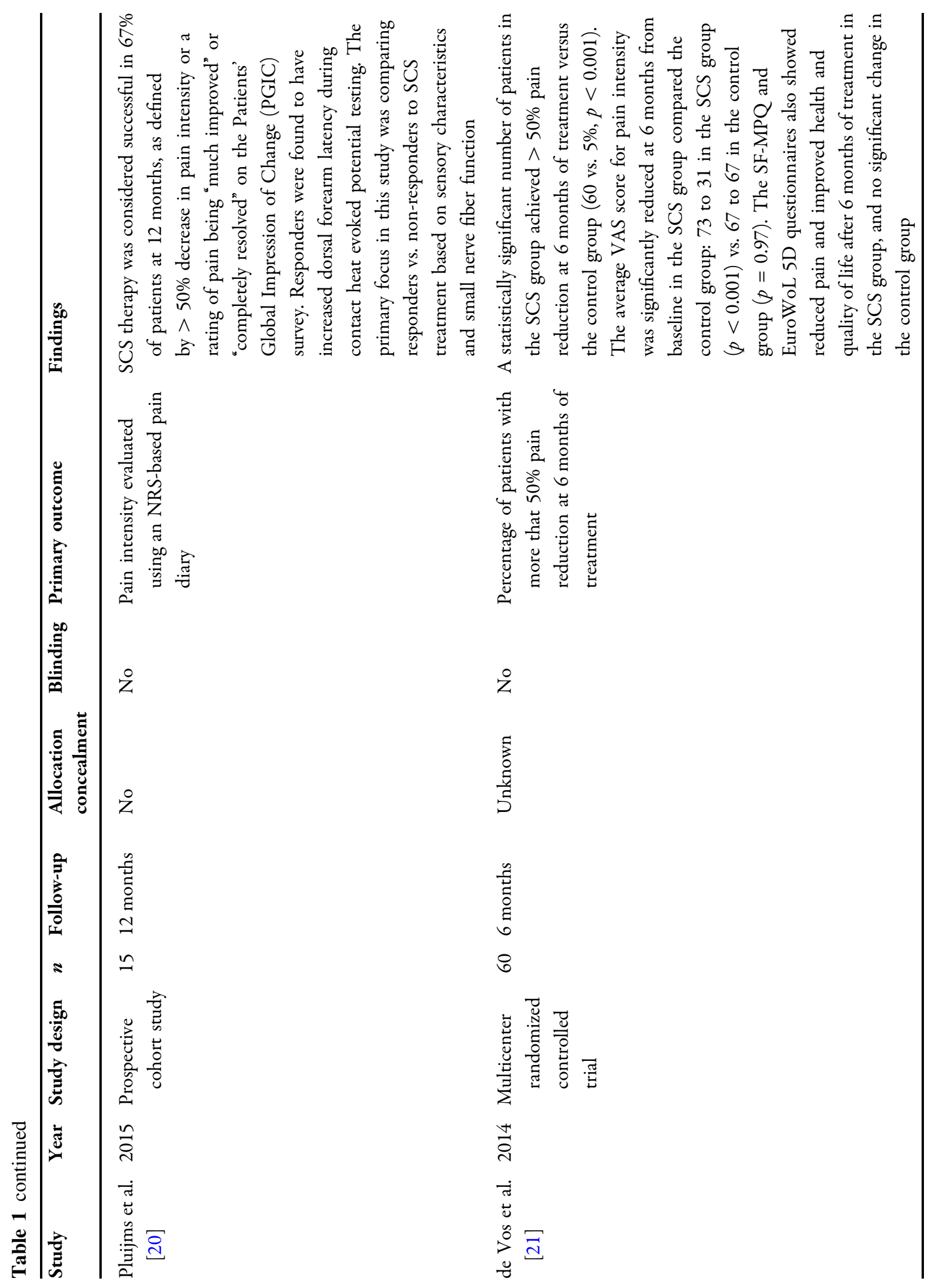




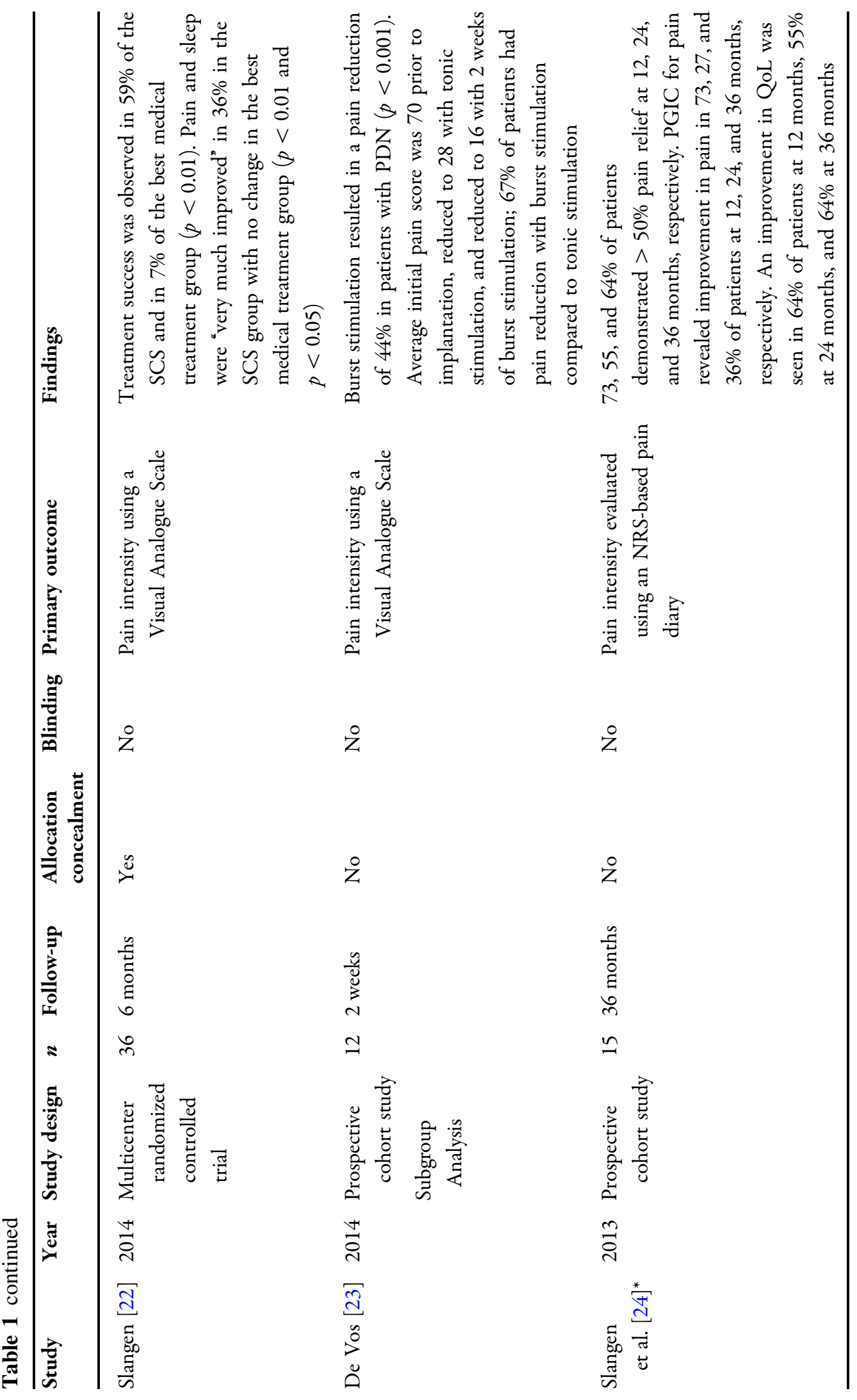




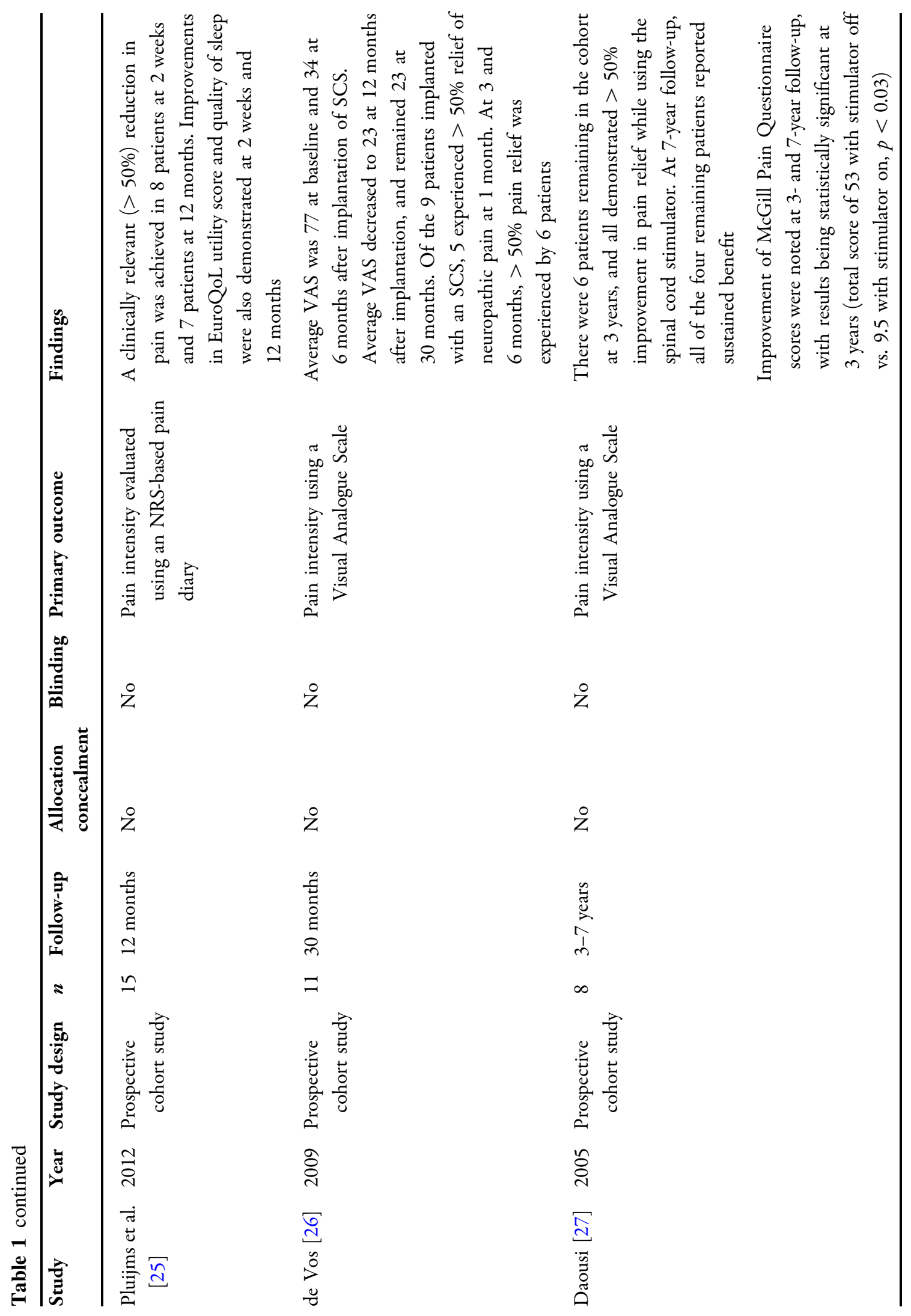




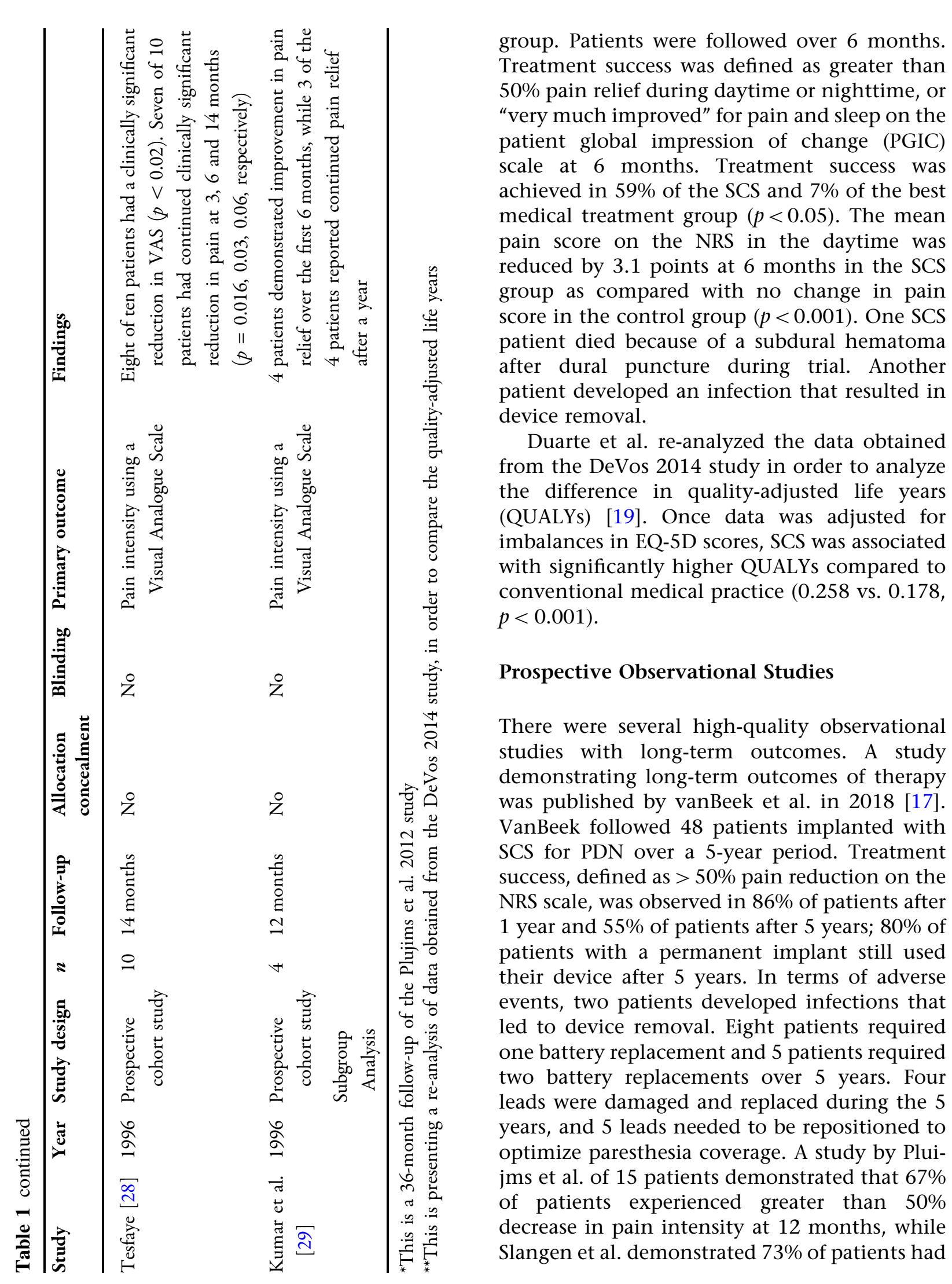




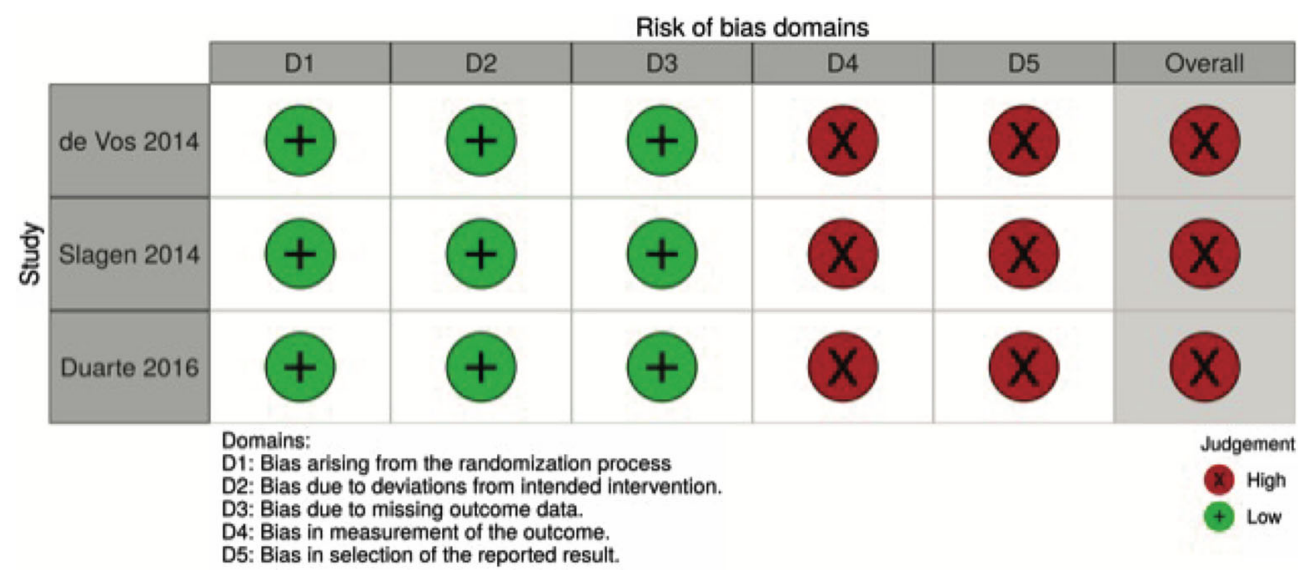

Fig. 2 Analysis of risk of bias using the Cochrane risk of bias 2 (RoB-2) tool

greater than $50 \%$ decrease in pain intensity at 12 months (though this dropped to $36 \%$ of patients at 36 months) [20, 24]. Daousi et al. demonstrated continued (greater than 50\%) pain relief in all patients remaining in their cohort after 7 years [27].

\section{Risk of Bias Assessment}

A risk-of-bias assessment was completed for all randomized control trials using the Revised Cochrane risk-of-bias tool for randomized trials (RoB 2) [15]. Studies were assessed across multiple domains per the specifications of the RoB 2 tool (Fig. 2). These domains included assessment of bias arising from the randomization process, bias due to deviations from the intended intervention, bias due to missing outcome data, bias in measurement of the outcome, and bias in selection of the reported result. The article by de Vos et al. [21] was not found to have significant risk of bias due to randomization, deviation from intervention, or missing data. However, a risk of bias was determined to be present due the method of measurement of the outcome. This is because the study was not double-blinded to spinal cord stimulator device implantation. It also was found to have high risk of bias due to selection of the reported result. In this study, there were multiple eligible analysis of the data, and the primary outcome reported was selected by converting a continuously scaled outcome measurement (in this case, pain level on a visual analogue scale) to categorical data with a specific cutoff point (in this case, percent of patients with 50\% improvement in pain). This confers a high risk of bias in the reported results. Because it had an increased risk of bias in two of the five domains, it was judged to have an overall high risk of bias. The article by Duarte et al. [19] was a re-analysis of de Vos study, and therefore was determined to have an elevated risk of bias for the same reasons.

The article by Slangen et al. [22] was similarly found to have an elevated risk of bias. Upon analysis with the RoB 2 tool, it was not found to have significant risk of bias due to randomization, deviation from intervention, or missing data. A risk of bias was determined to be present due the method of measurement of the outcome because the study was not double-blinded to spinal cord stimulator device implantation. It also was found to have high risk of bias due to selection of the reported result. In this study, there were multiple eligible analysis of the data, and the primary outcome reported was selected by converting a continuously scaled outcome measurement (in this case, Visual Analogue Scale) to categorical data with a specific cutoff point (in this case, percent of patients with $50 \%$ improvement in pain during daytime or nighttime or a response of "(very) much improved" for pain and sleep on the patient global impression of change scale at 6 months). This confers a high risk of bias in the reported results. Because it had an increased risk of bias 
in two of the five domains, it was judged to have an overall high risk of bias.

\section{DISCUSSION}

A review of the current literature regarding spinal cord stimulation for painful diabetic neuropathy revealed 14 studies that met our inclusion criteria. Two of these studies were randomized controlled trials with 6-month follow-up [21, 22], one of these studies provided additional analysis of the randomized controlled trial quality of life data [19], and the remainder were prospective observational studies. In the two randomized controlled trials analyzed, there was a clinically and statistically significant improvement in lower extremity pain and quality of life in patients who received spinal cord stimulation therapy [19, 21, 22]. All observational studies examined also demonstrated significant improvement in pain (see Table 1).

In the randomized controlled trial by de Vos et al., the adverse events related to the implantation procedure included pain due to the implanted pulse generator, electrode lead migration, infection, and bleeding complication. Slangen et al. reported one case of infection, as well as one SCS patient death due to subdural hematoma after dural puncture during the trial. A full literature search was conducted by the authors, and this was determined to be a very rare complication of device placement (only one other, non-fatal case of subdural hematoma was reported in the literature [30]).

In both randomized controlled trials, patients were excluded if they had significant symptoms of PDN in the upper extremities, which is a natural progression of the disease. Therefore, it is unclear based on this data if SCS will be as effective for PDN of the upper extremities.

Our systematic review demonstrated a lack of high-quality long-term data regarding SCS for PDN. The duration of follow-up for both randomized controlled trials analyzed was only 6 months, which may not have been adequate to assess the long-term effectiveness of this therapy. However, there were several high-quality observational studies that demonstrated longterm success of the therapy. A prospective observational study by vanBeek demonstrated that the improvement in pain is sustained by the majority of patients at 5 years [17]. These results were comparable to other prospective studies on SCS in neuropathic pain conditions like CRPS- 1 and FBSS [31, 32]. Limitations of this study included lack of randomization and relatively small sample size. Slangen et al. [24] also published long-term data, which demonstrated that $64 \%$ of patients demonstrated > $50 \%$ pain relief at 36 months.

All randomized controlled trials analyzed were determined to have a significant risk of bias (see Fig. 1). In these trials, no attempt was made to conceal the treatment arm to which each patient was allocated (trials were open-label). This introduced a significant risk of bias because outcomes in these studies are based on subjective patient-reported responses, and therefore could be significantly affected by lack of blinding.

In general, the observational studies analyzed in our systematic review echoed the results of the randomized controlled trials and generally support spinal cord stimulation for PDN. The most significant of these studies was a prospective cohort study conducted by van Beek et al. [17], which found that 55\% of patients continued to demonstrate greater than $50 \%$ pain relief after 5 years of spinal cord stimulation use. This was consistent with the results of multiple other studies analyzed, which all demonstrated significant and sustained pain relief up to 7 years after initial implantation [16, 17, 20, 24].

At the time searches were conducted for this systematic review, Mekhail et al. [33] was conducting a multicenter randomized controlled trial of 216 subjects with PDN assigned to receive $10-\mathrm{kHz}$ spinal cord stimulation versus conventional medical management (SENZAPDN). This trial will have 24-month follow-up. It is expected that the results of this study will contribute significantly to the body of evidence regarding spinal cord stimulation for PDN.

Finally, although impossible to make conclusive statements based on this review in terms of which PDN patients may benefit most from 
spinal cord stimulation, there are some notable findings. For example, the majority of patients were over the age of 50 (mean age ranged from 51 to 59.9), were more likely to have type 2 diabetes vs. type 1 and had diabetes greater than 10 years (ranging from 12 to 17 years). Furthermore, patients tended to have diabetic related pain for greater than 5 years (mean 5-7 years) at the time of SCS placement. The majority of patients had tried and failed multiple neuropathic medications and had a VAS greater than 5. Although it was not consistently included in these studies, A1C for these patients did seem to be under 10. For example, in the RCT by Slangen et al. the mean $\mathrm{A} 1 \mathrm{C}$ was 8.3 in the treatment group. Although it is difficult to extrapolate these observations, we can generally say that target patients seem to be older, have had painful diabetic neuropathy for many years, failed multiple medications, and did not have critically uncontrolled diabetes at the time of SCS placement. These observations may help with guidance in terms of when to consider SCS in painful diabetic peripheral neuropathy patients although further studies are needed for more definitive recommendations [16, 17, 19-29].

There are several limitations to this review. First, there is significant heterogeneity in the studies and limited RCT data. Many of the studies included were observational in nature and have the inherent biases that come with these types of studies. That said, the observational studies did support the RCT findings and hence strengthen those results. There were also variable or unclear types of stimulation used in the studies. It is uncertain whether high frequency vs. low frequency vs. burst stimulation is superior, and this potential variation in efficacy could affect study findings. Finally, some of the observational studies did not clearly state statistical significance. We did include these studies, as we believe they still offered useful data and add to the overall findings.

In summary, treatment of PDN with SCS demonstrated success in several high-quality multi-center randomized controlled trials. Based on these results, spinal cord stimulation can be recommended for patients with refractory pain due to diabetic neuropathy. However, the risk of bias due to the unblinded design of the studies as well as the limited duration of follow-up is important to acknowledge, and patient's expectations regarding duration of relief should be addressed prior to initiation of therapy.

\section{CONCLUSIONS}

Our review assessing spinal cord stimulation for peripheral diabetic neuropathy demonstrates evidence that it is an effective and safe option for treatment. However, further high-quality research, including a large-scale randomized controlled trial is warranted and would add to the current evidence.

\section{ACKNOWLEDGEMENTS}

Funding. No funding or sponsorship was received for this study or publication of this article.

Authorship. All named authors meet the International Committee of Medical Journal Editors (ICMJE) criteria for authorship for this article, take responsibility for the integrity of the work as a whole, and have given their approval for this version to be published.

Authors' Contributions. Josianna V. Henson, MD-Full text screening of studies, writing and editing the manuscript, creation of tables and figures. Narayana C. Varhabhatla, MD-Title and abstract screening, substantial edits to the manuscript. Zvonimir Bebic, MDFull text screening of studies, substantial edits to the manuscript. Alan D. Kaye MD-Substantial edits and drafting of the manuscript. R. Jason Yong, MD-Substantial edits and drafting of the manuscript. Richard D. Urman, MDContributed to paper concept and design, substantial edits, and drafting of the manuscript. Justin S. Merkow, MD-Concept and design of the paper, title and abstract screening, resolution of conflicts to full text screening, substantial edits, and drafting of the manuscript. 
Disclosures. Josianna V. Henson, MD, Narayana C. Varhabhatla, MD, Zvonimir Bebic, MD, Alan D. Kaye, MD, and Justin S. Merkow, MD have nothing to disclose. Dr. Urmans conflicts to Medtronic, Merck, Pfizer, and AcelRx. R. Jason Yong, MD-Consultant with Nevro, Medtronic, Abbot, and Endo Pharmaceuticals.

Compliance with Ethics Guidelines. This article is based on previously conducted studies and does not contain any new studies with human participants or animals performed by any of the authors.

Open Access. This article is licensed under a Creative Commons Attribution-NonCommercial 4.0 International License, which permits any non-commercial use, sharing, adaptation, distribution and reproduction in any medium or format, as long as you give appropriate credit to the original author(s) and the source, provide a link to the Creative Commons licence, and indicate if changes were made. The images or other third party material in this article are included in the article's Creative Commons licence, unless indicated otherwise in a credit line to the material. If material is not included in the article's Creative Commons licence and your intended use is not permitted by statutory regulation or exceeds the permitted use, you will need to obtain permission directly from the copyright holder. To view a copy of this licence, visit http://creativecommons.org/licenses/by$\mathrm{nc} / 4.0 /$.

\section{REFERENCES}

1. Prevention C for DC. National diabetes statistics report, 2017. Atlanta. 2017.

2. Association AD. Economic costs of diabetes in the US in 2017. Diabetes Care. 2018;41(5):917-28.

3. Schmader KE. Epidemiology and impact on quality of life of postherpetic neuralgia and painful diabetic neuropathy. Clin J Pain. 2002;18(6):350-4.

4. Snyder MJ, Gibbs LM, Lindsay TJ. Treating painful diabetic peripheral neuropathy: an update. Am Fam Physician. 2016;94(3):227-34.
5. Yang M, Qian C, Liu Y. Suboptimal treatment of diabetic peripheral neuropathic pain in the United States. Pain Med. 2015;16(11):2075-83.

6. Kemler MA, Barendse GA, Van Kleef M, et al. Spinal cord stimulation in patients with chronic reflex sympathetic dystrophy. N Engl J Med. 2000;343(9): 618-24.

7. Kemler MA, De Vet HC, Barendse GA, Van den Wildenberg FA, Van Kleef M. Effect of spinal cord stimulation for chronic complex regional pain syndrome Type I: five-year final follow-up of patients in a randomized controlled trial. J Neurosurg. 2008;108(2):292-8.

8. Kumar K, Taylor RS, Jacques L, et al. Spinal cord stimulation versus conventional medical management for neuropathic pain: a multicentre randomised controlled trial in patients with failed back surgery syndrome. Pain. 2007;132(1-2):179-88.

9. North RB, Kumar K, Wallace MS, et al. Spinal cord stimulation versus re-operation in patients with failed back surgery syndrome: an international multicenter randomized controlled trial (EVIDENCE study). Neuromodulation Technol Neural Interface. 2011;14(4):330-6.

10. Joosten EA, Franken G. Spinal cord stimulation in chronic neuropathic pain: mechanisms of action, new locations, new paradigms. Pain. 2020;161(1): S104.

11. Deer T, Slavin KV, Amirdelfan K, et al. Success using neuromodulation with BURST (SUNBURST) study: results from a prospective, randomized controlled trial using a novel burst waveform. Neuromodulation Technol Neural Interface. 2018;21(1):56-66.

12. Kapural L, Yu C, Doust MW, et al. Novel $10-\mathrm{kHz}$ high-frequency therapy (HF10 therapy) is superior to traditional low-frequency spinal cord stimulation for the treatment of chronic back and leg pain: the SENZA-RCT randomized controlled trial. Anesthesiology. 2015;123(4):851-60.

13. Thomson SJ, Tavakkolizadeh M, Love-Jones S, et al. Effects of rate on analgesia in kilohertz frequency spinal cord stimulation: results of the PROCO randomized controlled trial. Neuromodulation Technol Neural Interface. 2018;21(1):67-76.

14. Frantzi K, Ananiadou S, Mima H. Automatic recognition of multi-word terms: the c-value/ncvalue method. Int J Digit Libr. 2000;3(2):115-30.

15. Sterne JA, Savović J, Page MJ, et al. RoB 2: a revised tool for assessing risk of bias in randomised trials. Bmj. 2019;366. 
16. Galan V, Scowcroft J, Chang P, et al. 10-kHz spinal cord stimulation treatment for painful diabetic neuropathy: results from post-hoc analysis of the SENZA-PPN study. Pain Manag. 2020;10(5): 291-300.

17. van Beek M, Geurts JW, Slangen R, et al. Severity of neuropathy is associated with long-term spinal cord stimulation outcome in painful diabetic peripheral neuropathy: five-year follow-up of a prospective two-center clinical trial. Diabetes Care. 2018;41(1): 32-8.

18. Denisova NP, Rogov DY, Rzaev DA, Khabarova EA, Dmitriev AB. Spinal cord stimulation in the treatment of chronic pain syndromes. Zh Vopr Neirokhir Im NN Burdenko. 2016;80(2):47-52.

19. Duarte RV, Andronis L, Lenders MW, de Vos CC. Quality of life increases in patients with painful diabetic neuropathy following treatment with spinal cord stimulation. Qual Life Res. 2016;25(7): 1771-7.

20. Pluijms WA, Slangen R, van Kleef M, Joosten EA, Reulen JP. Increased contact heat evoked potential stimulation latencies in responders to spinal cord stimulation for painful diabetic polyneuropathy. Neuromodulation Technol Neural Interface. 2015;18(2):126-32.

21. de Vos CC, Meier K, Zaalberg PB et al. (2014) Spinal cord stimulation in patients with painful diabetic neuropathy: a multicentre randomized clinical trial. PAIN 155(11):2426-2431

22. Slangen R, Schaper NC, Faber CG, et al. Spinal cord stimulation and pain relief in painful diabetic peripheral neuropathy: a prospective two-center randomized controlled trial. Diabetes Care. 2014;37(11):3016-24.

23. de Vos CC, Bom MJ, Vanneste S, Lenders MW, De Ridder D. Burst spinal cord stimulation evaluated in patients with failed back surgery syndrome and painful diabetic neuropathy. Neuromodulation Technol Neural Interface. 2014;17(2):152-9.

24. Slangen R, Pluijms WA, Faber CG, Dirksen CD, Kessels AGH, Van Kleef M. Sustained effect of spinal cord stimulation on pain and quality of life in painful diabetic peripheral neuropathy. $\mathrm{Br} \mathrm{J}$ Anaesth. 2013;111(6):1030-1.
25. Pluijms WA, Slangen R, Bakkers M, et al. Pain relief and quality-of-life improvement after spinal cord stimulation in painful diabetic polyneuropathy: a pilot study. Br J Anaesth. 2012;109(4):623-9.

26. de Vos CC, Rajan V, Steenbergen W, van der Aa HE, Buschman HP. Effect and safety of spinal cord stimulation for treatment of chronic pain caused by diabetic neuropathy. J Diabetes Complications. 2009;23(1):40-5.

27. Daousi C, Benbow SJ, MacFarlane IA. Electrical spinal cord stimulation in the long-term treatment of chronic painful diabetic neuropathy. Diabet Med. 2005;22(4):393-8.

28. Tesfaye S, Watt J, Benbow SJ, Pang KA, Miles J, MacFarlane IA. Electrical spinal-cord stimulation for painful diabetic peripheral neuropathy. Lancet. 1996;348(9043):1698-701.

29. Kumar K, Toth C, Nath RK. Spinal cord stimulation for chronic pain in peripheral neuropathy. Surg Neurol. 1996;46(4):363-9.

30. Chiravuri S, Wasserman R, Chawla A, Haider N. Subdural hematoma following spinal cord stimulator implant. Pain Physician. 2008;11(1):97-101.

31. Geurts JW, Smits H, Kemler MA, Brunner F, Kessels AG, van Kleef M. Spinal cord stimulation for complex regional pain syndrome type I: a prospective cohort study with long-term follow-up. Neuromodulation Technol Neural Interface. 2013;16(6): 523-9.

32. Kumar K, Taylor RS, Jacques L, et al. The effects of spinal cord stimulation in neuropathic pain are sustained: a 24-month follow-up of the prospective randomized controlled multicenter trial of the effectiveness of spinal cord stimulation. Neurosurgery. 2008;63(4):762-70.

33. Mekhail NA, Argoff CE, Taylor RS, et al. High-frequency spinal cord stimulation at $10 \mathrm{kHz}$ for the treatment of painful diabetic neuropathy: design of a multicenter, randomized controlled trial (SENZAPDN). Trials. 2020;21(1):87.

34. Moher D, Liberati A, Tetzlaff J, Altman DG, Group P. Preferred reporting items for systematic reviews and meta-analyses: the PRISMA statement. PLoS Med. 2009;6(7):e1000097. 